\title{
COVID-19 in a Patient Treated for Granulomatosis with Polyangiitis: Persistent Viral Shedding with No Cytokine Storm
}

\author{
Pascale Daniel $^{1}$, Marc Raad ${ }^{2}$, Rami Waked ${ }^{3}$, Jacques Choucair ${ }^{3}$, Moussa Riachy², Fady Haddad ${ }^{1}$ \\ ${ }^{1}$ Department of Internal Medicine and Clinical Immunology, Hotel-Dieu de France University Hospital, Saint Joseph University, Beirut, Lebanon \\ ${ }^{2}$ Department of Pulmonary and Critical Care Medicine, Hotel-Dieu de France University Hospital, Saint Joseph University, Beirut, Lebanon \\ ${ }^{3}$ Department of Infectious Diseases, Hotel-Dieu de France University Hospital, Saint Joseph University, Beirut, Lebanon
}

\section{Doi: 10.12890/2020_001922 - European Journal of Case Reports in Internal Medicine - ๑ EFIM 2020}

Received: $27 / 07 / 2020$

Accepted: 04/08/2020

Published: $24 / 09 / 2020$

\begin{abstract}
How to cite this article: Daniel P, Raad M, Waked R, Choucair J, Riachy M, Haddad F. COVID-19 in a patient treated for granulomatosis with polyangiits: persistent viral shedding with no cytokine storm. EJCRIM 2020;7: doi:10.12890/2020_001922.
\end{abstract}

Conflicts of Interests: The Authors declare that there are no competing interests.

This article is licensed under a Commons Attribution Non-Commercial 4.0 License

\section{ABSTRACT}

Introduction: The coronavirus disease COVID-19 is considered a pandemic disease that has developed rapidly all over the world. As of today, it is unclear whether immunosuppression confers an increased risk for pulmonary complications, or conversely, whether it can be a protective factor with respect to a cytokine storm.

Case description: We report the case of a 55-year-old male patient with granulomatosis with polyangiitis treated with rituximab who was infected with COVID-19 pneumonia. To the best of our knowledge, only 1 case has been reported in the literature with similar characteristics. The patient had a non-classic evolution of clinical symptoms with persistent fever and viral shedding, in addition to a negative serology. Conclusion: This case emphasizes the management and immunity response to COVID-19 pneumonia in such patients. Data are still needed regarding patients who have prolonged B-cell depletion, which may put the patient at a higher risk for reinfection.

\section{LEARNING POINTS}

- Demonstration of the immunity response to COVID-19 pneumonia in an immunosuppressed patient.

- To highlight the management and evolution of such rare cases during this pandemic.

\section{KEYWORDS}

COVID-19, SARS-CoV-2, granulomatosis with polyangiitis, rituximab

\section{INTRODUCTION}

The outbreak of the new coronavirus disease COVID-19 in Wuhan, China in December 2019, caused by severe acute respiratory syndrome coronavirus 2 (SARS-CoV-2), has since developed rapidly to become a pandemic disease as declared by the World Health Organization on March 12, 2020 with a mortality rate of approximately 7\% as of April 27, 2020[1].

As of today, risk factors for a poor outcome include advanced age, male sex and the presence of comorbidities, especially obesity, diabetes and heart and lung diseases ${ }^{[2]}$. These were also applicable to the SARS outbreak that emerged in 2002, and the Middle East respiratory syndrome in 2012, both of which had the same risk factors for a poor outcome. Immunosuppressed status was not found to be a risk factor among these ${ }^{[3]}$. As is well known, immunosuppressive medications can alter humoral and cell-mediated immunity, by increasing the risk of severe infections caused by viral agents, as well as superimposed bacterial infections ${ }^{[4,5]}$. 
This does not seem to be the case for infections caused by the coronavirus family known to date. Some attribute this to an overactive immune response causing clinical deterioration, which is impaired in these immunocompromised patients ${ }^{[4,6]}$.

However, this does not mean that we should not raise concern for this group of patients, and we must have a high level of vigilance and strict follow-up. We still do not have enough evidence to assess the risk of respiratory or life-threatening complications from SARS-CoV-2 infection in this immunosuppressed population. Reviewing the literature, there are insufficient data regarding the management and treatment of SARS-CoV-2 patients with immune diseases. We report the case of a COVID-19 patient with granulomatosis with polyangiitis (GPA), treated with corticosteroids and rituximab (chimeric murine/human anti-CD20 monoclonal antibody). ANCA-associated vasculitis and more specifically, GPA, can involve the respiratory tract, making these patients more vulnerable to COVID-19-related pulmonary complications. Treating these patients with rituximab will result in prolonged B-cell depletion with impairment of pre-existing humoral immunity ${ }^{[5]}$.

\section{CASE DESCRIPTION}

We present the case of a 55-year-old man who was treated for GPA since 1994 (episcleritis, arthralgia, pulmonary involvement with alveolar haemorrhage, renal involvement and pachymeningitis). He had since received many immunosuppressive agents such as cyclophosphamide, azathioprine and methotrexate. He was on $4 \mathrm{mg}$ of prednisone in addition to a periodic 6-monthly infusion of $500 \mathrm{mg}$ of rituximab as a maintenance therapy since 2005 (his next infusion was planned for April 2020). He was also on a prophylactic trimethoprim/sulfamethoxazole treatment ( 3 times weekly). His main comorbidities included hypertension treated with atenolol, irbesartan and hydrochlorothiazide, and type 2 diabetes treated with glimepiride. He was known to have stage 3B chronic kidney disease.

On March 21, 2020 the patient developed respiratory symptoms with a dry cough, followed 3 days later by a $40^{\circ} \mathrm{C}$ persistent fever. $\mathrm{He}$ was admitted to our hospital on March 28 (Day 0). His clinical examination was normal. COVID-19 pneumonia was diagnosed on that day after a positive nasal reverse transcription PCR (RT-PCR) test, and chest CT showing bilateral mild ground-glass opacities with a minimal degree of involvement of $10 \%$ (Fig. 1).

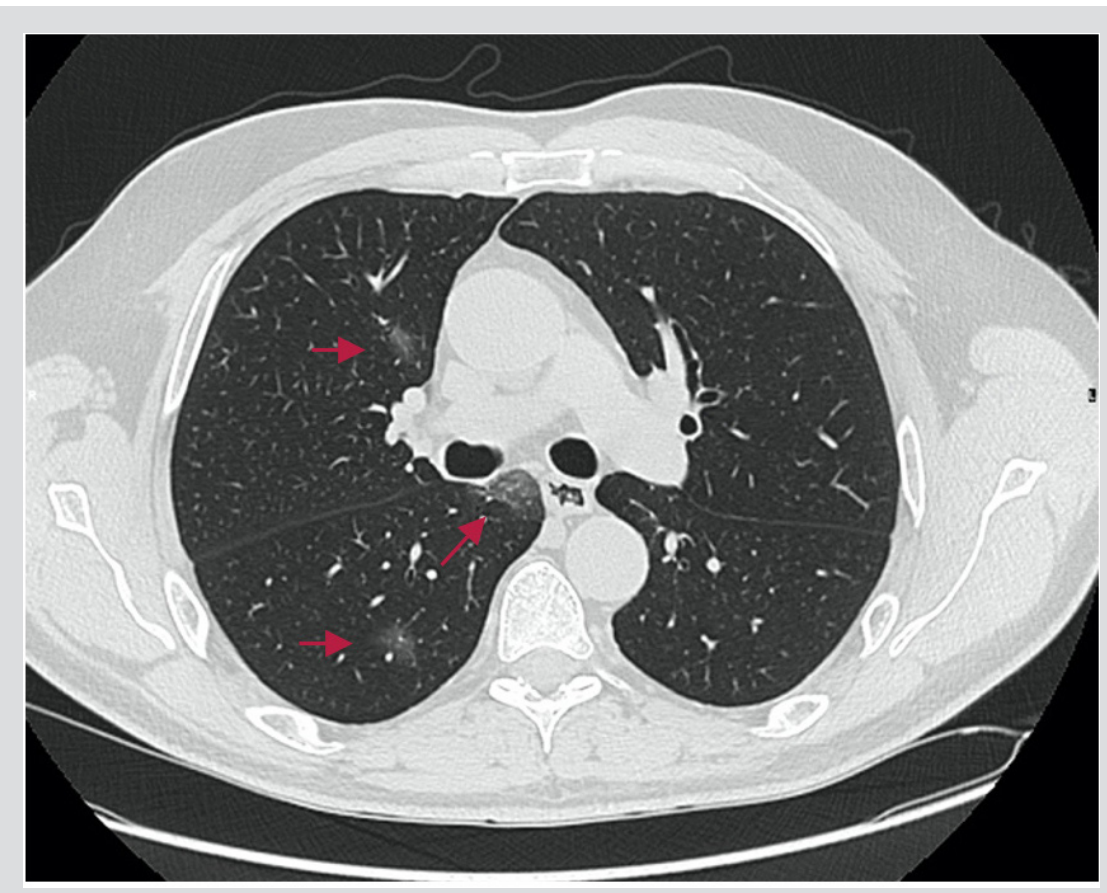

Figure 1. Chest CT on Day 0 showing bilateral mild ground-glass opacities (red arrows) with a minimal degree of involvement (10\%)

On the day of admission, the patient was stable with a National Early Warning Score 2 (NEWS2) COVID-19 score of 0. He was treated according to our hospital protocol with 7 days of hydroxychloroquine (400 mg bid on Day 1 then $200 \mathrm{mg}$ tid), 5 days of azithromycin (500 mg on Day 1 then 250 mg od for 4 days), 7 days of zinc ( 2 tabs per day), as well as pitavastatin ( 2 mg per day for 14 days). Despite the treatment, the patient remained highly febrile, with a profuse watery diarrhoea treated with diosmectite after eliminating an infectious cause. He was haemodynamically stable with no need for oxygen therapy. On Day 7 of hospitalization (Day 14 from the appearance of symptoms), with the patient remaining febrile $\left(40^{\circ} \mathrm{C}\right)$, a laboratory work-up was done. Blood cultures, qualitative Cytomegalovirus PCR testing of blood samples, brucellosis and typhoid fever serologies all came back negative. Lopinavir 400 mg/ritonavir 100 mg bid was started (following our 
hospital protocol) but was stopped 7 days later because of severe diarrhoea. On April 8, 2020 (Day 12), the patient was still febrile, without a progression in respiratory symptoms (NEWS2 score of 2), and all cultures remained negative. A full body CT scan carried out on that day showed a significant progression of the bilateral ground-glass opacities with 60\% involvement of pulmonary fields (Fig. 2).

The evolution of the patient's absolute lymphocyte count, C-reactive protein levels and the neutrophil to lymphocyte ratio during his hospitalization is shown in Fig. 3

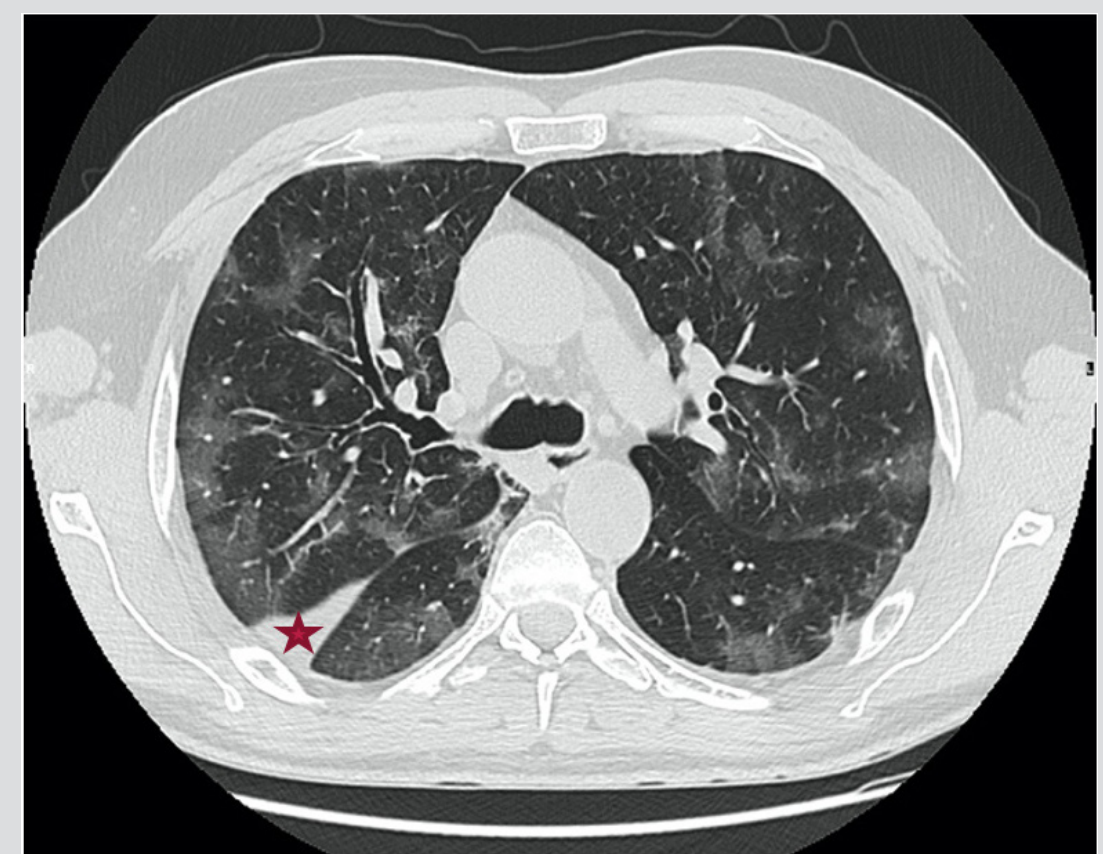

Figure 2. Chest CT on Day 12 showing bilateral mild ground-glass opacities with a degree of involvement of more than $60 \%$ with peripheral linear tracts (red star)

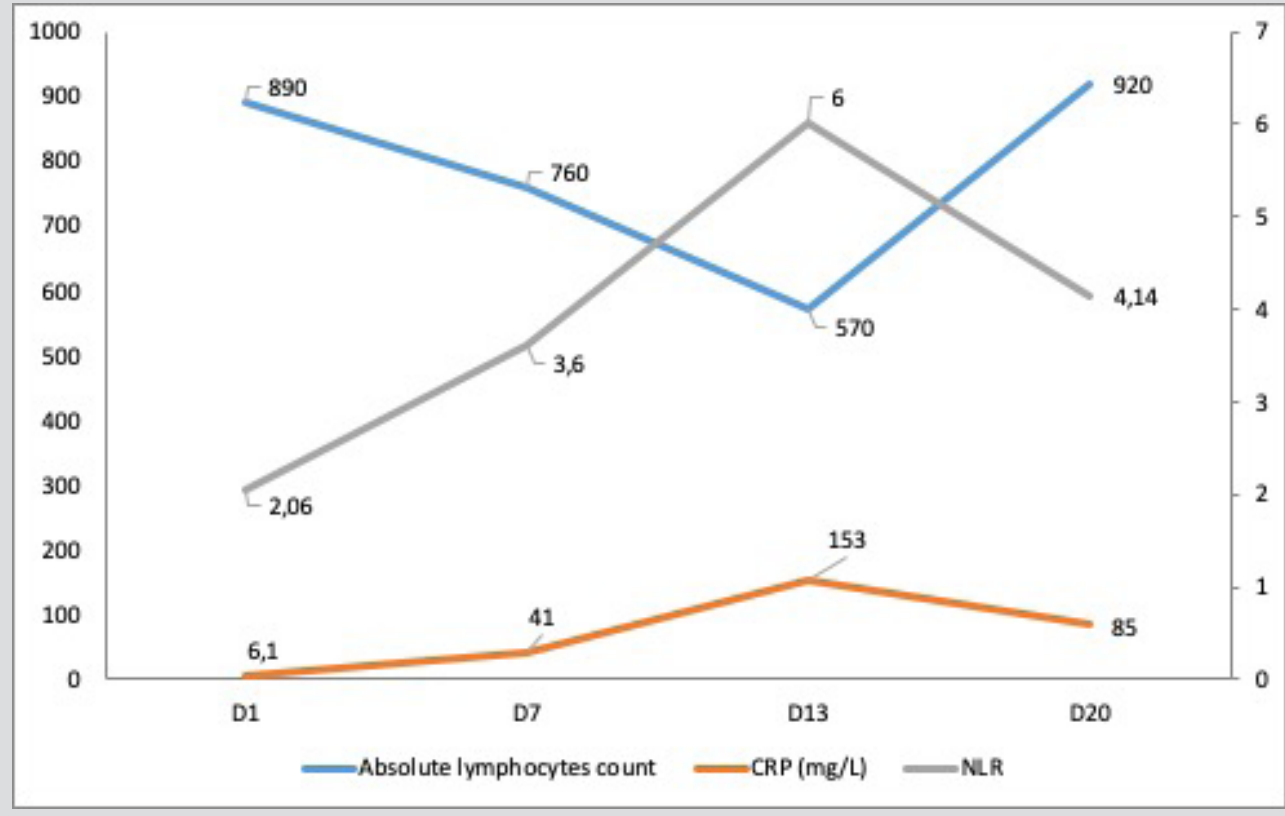

Figure 3. Evolution of the absolute lymphocyte count, $\mathrm{C}$-reactive protein (CRP) levels and the neutrophil to lymphocyte ratio (NLR) over days of hospitalization

In view of the immunocompromised status of this patient, and the impossibility of differentiating between an opportunistic infection and the disease itself on CT images, a broad-spectrum antibiotic (piperacillin/tazobactam) was started, and a second course of hydroxychloroquine was initiated. On Day 13, he developed lymphangitis confirmed by venous Doppler ultrasound revealing superficial venous thrombosis of the left cephalic vein. A glycopeptide was added, and therapeutic heparin therapy was initiated. Despite broad-spectrum antibiotics, the 
patient remained febrile until Day 19 of hospitalization (Day 26 after the onset of symptoms). On Day 18, an RT-PCR test was repeated and remained positive. On Day 20, the fever as well as the patient's other symptoms disappeared, without any change in treatment, and before continuing our investigations with another chest CT scan and bronchoscopy with scheduled bronchoalveolar lavage, as discussed in a multidisciplinary meeting. After 48 hours of complete apyrexia, the patient was discharged home with specific instructions on home isolation. The patient improved clinically and became completely asymptomatic but PCR testing remained positive for approximately 2 months (the first negative RT-PCR test was on June 6, 2020). The patient also had a COVID-19 serology test with IgG and IgM antibody negativity $(0.1 \mathrm{AU} / \mathrm{ml}$ and $0.6 \mathrm{AU} / \mathrm{ml}$, respectively, using CLIA or chemiluminescence immunoassay). It should be noted that his absolute CD4 count in peripheral blood was $564 / \mathrm{mm}^{3}$.

\section{DISCUSSION}

Several differential diagnoses were proposed during the clinical progression of this case: is it a pulmonary superimposed resistant infection not covered by our antibiotics? Is it a septic thrombophlebitis? Is the fever only related to COVID-19 infection? Or is the fever related to a flare-up of the vasculitis, especially as rituximab treatment was postponed?

This report describes, to the best of our knowledge, the second case of a patient treated with rituximab and glucocorticoids for an ANCAassociated vasculitis (GPA). A previous publication reported on a patient who experienced respiratory failure and needed intubation, emphasizing the highly progressive worsening of her clinical condition compared to other case series, and her rapid improvement ${ }^{[7]}$. With respect to our clinical case, we noticed a radiological progression and a persistent high-grade fever 26 days after symptoms appeared, without the need for high oxygen supplementation with a persistent low-risk NEWS2 COVID-19 score. We hypothesize that the patient's B-cell depletion and reduced immune response may have played a favourable role in preventing an overly active reaction and a cytokine storm ${ }^{[8]}$. However, with persistent positive RT-PCR testing almost 2 months after the onset of symptoms, and a negative serology, we can assume that the rituximab administered within a repeated dosing strategy may have impaired the immunological response of seroconversion ${ }^{[9]}$. Despite our patient having many other risk factors for a poor COVID-19 prognosis, such as being male and having diabetes mellitus, as well as having other risk factors for opportunistic infections (glucocorticoid treatment over a long period, treatment with other immunosuppressive drugs), he showed a relatively indolent pattern with respect to the course of symptoms during his hospital stay. In addition, rituximab compromises the immunization response and alters pre-existing circulating antibodies and memory B lymphocytes, putting the patient at a higher risk of reinfection, although the duration and effectiveness of antibody protection is not yet known.

\section{CONCLUSION}

At this point of the pandemic outbreak, we lack data on the management of such cases. More studies are needed regarding the management, prognosis and immunity response of such immunocompromised patients treated with immunosuppressive agents, specifically rituximab, for an underlying auto-immune/inflammatory disease.

\section{REFERENCES}

1. Worldometer [Internet]. Coronavirus updates (live): 3,029,746 cases and 209,244 deaths from the COVID-19 virus pandemic. Available from: https://www.worldometers.info/ coronavirus/ (accessed 27 April 2020).

2. Zheng Z, Peng F, Xu B, Zhao J, Liu H, Peng J, et al. Risk factors of critical \& mortal COVID-19 cases: a systematic literature review and meta-analysis. J Infect 2020;81:e16-e25.

3. de Wit E, van Doremalen N, Falzarano D, Munster V. SARS and MERS: recent insights into emerging coronaviruses. Nat Rev Microbiol 2016;14(8):523-534.

4. D'Antiga L. Coronaviruses and immunosuppressed patients: the facts during the third epidemic. Liver Transpl 2020;26:832-834.

5. Marco H, Smith RM, Jones RB, Guerry MJ, Catapano F, Burns S, et al. The effect of rituximab therapy on immunoglobulin levels in patients with multisystem autoimmune disease. BMC Musculoskelet Disord 2014;15:178.

6. Shi Y, Wang Y, Shao C, Huang J, Gan J, Huang X, et al. COVID-19 infection: the perspectives on immune responses. Cell Death Differ 2020;27(5):1451-1454.

7. Guilpain P, Le Bihan C, Foulongne V, Taourel P, Pansu N, Maria ATJ, et al. Rituximab for granulomatosis with polyangiitis in the pandemic of covid-19: lessons from a case with severe pneumonia. Ann Rheum Dis. Epub 2020 Apr 20. doi: 10.1136/annrheumdis-2020-217549.

8. Monti S, Balduzzi S, Delvino P, Bellis E, Quadrelli VS, Montecucco C. Clinical course of COVID-19 in a series of patients with chronic arthritis treated with immunosuppressive targeted therapies. Ann Rheum Dis 2020;79(5):667-668

9. Bouadma L, Lescure FX, Lucet JC, Yazdanpanah Y, Timsit JF. Severe SARS-CoV-2 infections: practical considerations and management strategy for intensivists. Intensive Care Med 2020;46(4):579-582. 